\title{
ERRATUM
}

Eberhard Gischler • Anthony J. Lomando

\section{Offshore sedimentary facies of a modern carbonate ramp, Kuwait, northwestern Arabian-Persian Gulf}

Published online: 18 June 2005

(C) Springer-Verlag 2005

\section{Facies (2005) 50:443-462}

In this contribution, Table 8 was incorrectly rendered in the print version and in the pdf version online (the html version online is correct).

The table should be rendered as follows:

Table 8
Table 8 Correlation matrix of isotope and mineralogy data (upper value is $\mathrm{r}$; statistically significant correlation at $p<0.05$ )

\begin{tabular}{lllllll}
\hline & LMC & ARA & DOL & HMC & $\delta 13 C$ & $\delta 180$ \\
\hline LMC & 1 & & & & \\
& $\mathrm{p}<.000$ & & & & \\
& & & & & \\
ARA & -.93246 & 1 & & & \\
& $\mathrm{p}<0.000$ & $\mathrm{p}<.000$ & & & \\
& & & & & \\
DOL & .90218 & -.82611 & 1 & & \\
& $\mathrm{p}<0.000$ & $\mathrm{p}<0.000$ & $\mathrm{p}<.000$ & & \\
& & & & & \\
HMC & .51983 & -.79166 & .39547 & 1 & \\
& $\mathrm{p}<0.000$ & $\mathrm{p}<0.000$ & $\mathrm{p}<0.000$ & $\mathrm{p}<.000$ & \\
& & & & & \\
$\delta 13 C$ & -.59433 & .72520 & -.43327 & -.72818 & 1 & \\
& $\mathrm{p}<0.000$ & $\mathrm{p}<0.000$ & $\mathrm{p}<0.000$ & $\mathrm{p}<0.000$ & $\mathrm{p}<.000$ & \\
& & & & & & \\
$\delta 180$ & -.54024 & .55785 & -.59234 & -.38271 & .55529 & 1 \\
& $\mathrm{p}<0.000$ & $\mathrm{p}<0.000$ & $\mathrm{p}<0.000$ & $\mathrm{p}<0.000$ & $\mathrm{p}<0.000$ & $\mathrm{p}<.000$ \\
\hline
\end{tabular}

The online version of the original article can be found at http://dx.doi.org/10.1007/s10347-004-0027-4.

E. Gischler $(\triangle)$

Geologisch-Paläontologisches Institut, Johann Wolfgang

Goethe-Universität,

Senckenberganlage 32,

60054 Frankfurt am Main, Germany

e-mail: gischler@em.uni-frankfurt.de

Tel.: +49-69-79825136

\section{A. J. Lomando}

ChevronTexaco, Kuwait Pouch,

93 Wigmore Street,

London, W1U 1HH, UK 